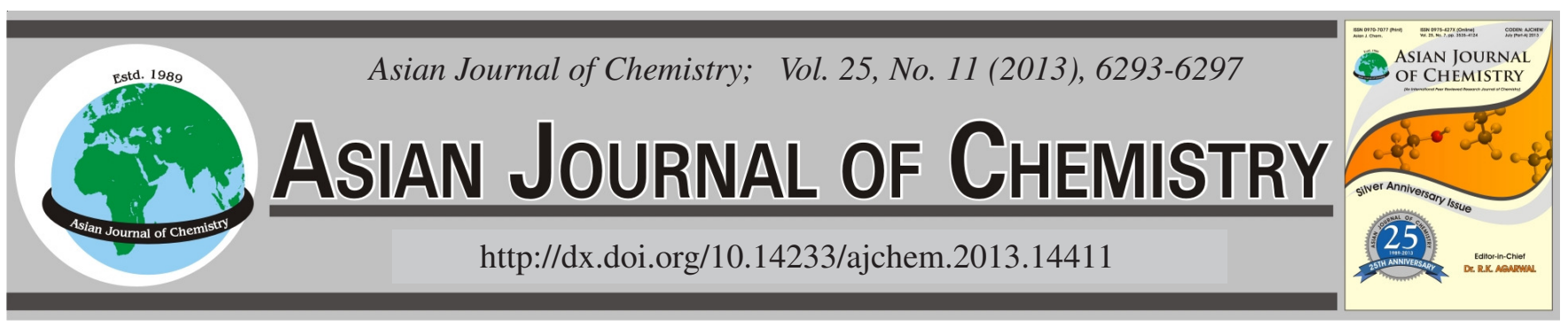

\title{
Analysis of Interfering Substances in the Measurement of Malondialdehyde Content in Plant Leaves
}

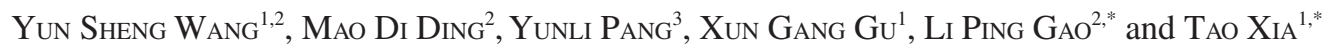

${ }^{1}$ Key Laboratory of Tea Biochemistry and Biotechnology, Ministry of Education in China, Anhui Agricultural University, Hefei, Anhui Province, P.R. China

${ }^{2}$ School of Life Sciences, Anhui Agricultural University, Hefei, Anhui Province, P.R. China

${ }^{3}$ Department of Mathematics, Xianyang Normal University, Xianyang, Shaanxi Province, P.R. China

*Corresponding author: Fax: +86 551 5785729; Tel: +86 551 5786232; E-mail: gaolp62@126.com; xiatao62@126.com

\begin{abstract}
The thiobarbituric acid reactive substances assay is an easy and quick assay for the assessment in plants of lipid peroxidation, in which malondialdehyde is derivatized. To analyze the applicability of this method, the malondialdehyde concentrations in the leaves of different plants were measured by the thiobarbituric acid method. For further separation of the interfering substances, fractional extraction and gas chromatography-mass spectrometry were used in this research. The results suggest that the lipid peroxidation is accurately measured by the thiobarbituric acid method with trichloroacetic acid as the extraction solution in most plants. However, the method was not suitable for measuring the malondialdehyde concentration in golden privet (Ligustrum $\times$ vicaryi) leaves. Negative malondialdehyde concentrations were obtained in golden privet leaves by this method.
\end{abstract}

Key Words: Gas chromatography-mass spectrometry, Interfering substance, Lipid peroxidation, Malondialdehyde, Thiobarbituric acid.

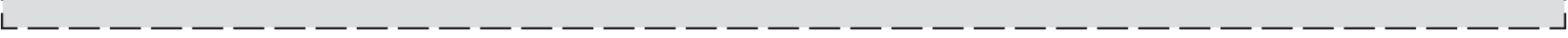

\section{INTRODUCTION}

In plants, the toxic effects of abiotic and biotic stresses include a decrease in photosynthetic activity ${ }^{1}$, a reduction in water and nutrient uptake, growth inhibition ${ }^{2,3}$, damage to proteins $^{4}$ and changes in lipid composition ${ }^{5}$ and lipid peroxidation $^{6}$. Lipid peroxidation has been suggested to be responsible for the developmental processes of plants, including the juvenile stages, production of volatile odors, senescence and formation of compounds such as jasmonic acid ${ }^{7}$. This process can be initiated by free radicals or enzymatic activities ${ }^{8}$. Therefore, the detection of lipid peroxidation is important in research into plant senescence and environmental stress.

Malondialdehyde (MDA), a naturally occurring product of lipid peroxidation, is the important indicator for the process 9 . The thiobarbituric acid (TBA) reactive substances assay is an easy and quick assay for the assessment of malondialdehyde concentration in plants. In this assay, malondialdehyde reacts with thiobarbituric acid to form a pink pigment, a condensation product of thiobarbituric acid and malondialdehyde in a 2:1 molar ratio, which has an absorption maximum at $532 \mathrm{~nm}^{10}$. In plant tissue, however, certain compounds (anthocyanins and carbohydrates) may interfere with measurements at this wavelength. Furthermore, the presence of barbituric acid impurities in the thiobarbituric acid reagent has been found to produce 1:1:1 TBA/MDA/barbituric acid and 2:1 barbituric acid/MDA adducts that absorb at 513 and $490 \mathrm{~nm}^{11}$, respectively.

The aim of this work is to analyze the applicability of the thiobarbituric acid method for measuring malondialdehyde concentrations in various plants. As a further check of the malondialdehyde extraction rate, two frequently used solutions, phosphate buffer and trichloroacetic acid, were compared. In golden privet (Ligustrum $\times$ vicaryi) leaves, substances were found that interfered with the measurement of malondialdehyde concentration using the thiobarbituric acid method. To separate the interfering substances, fractional extraction and gas chromatography-mass spectrometry were used.

\section{EXPERIMENTAL}

The functional and the senescent leaves of 12 plants grown at the botanical gardens of the Anhui Agricultural University (eastern China, $32^{\circ} \mathrm{N}$ ) were selected as the materials (Fig. 1). The samples were collected, placed immediately into an ice box, weighed, quickly frozen in liquid nitrogen and stored at $-80{ }^{\circ} \mathrm{C}$.

Extraction of malondialdehyde: Fresh functional or senescent leaves $(0.5 \mathrm{~g})$ from at least seven plants were taken 

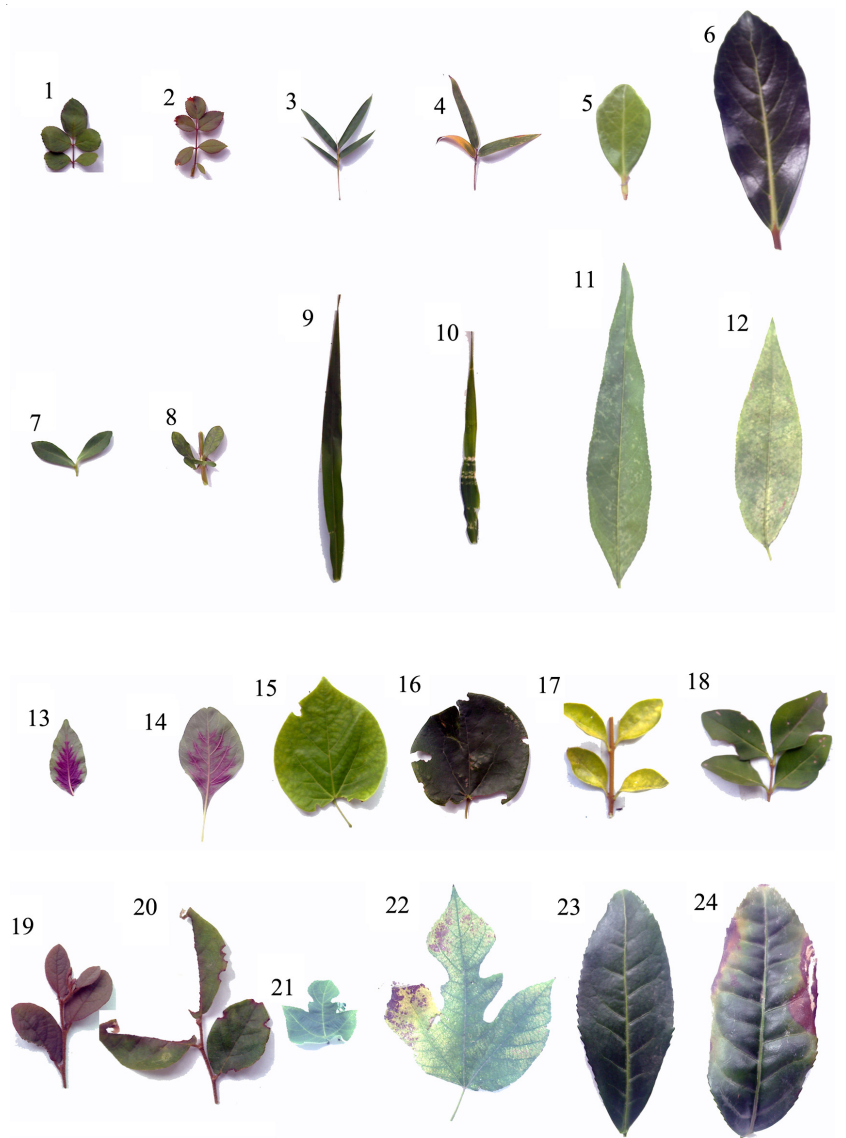

Fig. 1. Leaves of various plants sampled for the analysis of MDA concentrations. Leaves shown are: 1,2: multiflora rose (Rosa multiflora); 3,4: bamboo (Phyllostachys bambusoides); 5,6: sweet viburnum (Viburnum odoratissimum); 7,8: alligator weed (Alternanthera philoxeroides); 9,10: green bristlegrass (Setaria viridis); 11,12: peach tree (Prunus persica); 13,14: amaranth (Amaranthus gangeticus); 15,16: Chinese redbud (Cercis chinensis); 17,18: golden privet (Ligustrum $\times$ vicaryi); 19,20: red-flowering loropetalum (Loropetalum chindensevar); 21,22: paper mulberry (Broussonetia papyrifera); 23,24: tea plant (Camellia sinensis). For each species, odd numbers indicate functional leaves and even numbers indicate senescent leaves

and ground in $5 \mathrm{~mL}$ extraction solution (separately using 0.05 mol L ${ }^{-1} \mathrm{pH} 7.8$ phosphate buffer or $5 \%$ trichloroacetic acid as extraction solution), followed by centrifugation at $5000 \mathrm{~g}$ for $15 \mathrm{~min}$. The supernatant is the malondialdehyde extraction solution, which was stored at $4{ }^{\circ} \mathrm{C}$.

Measurement of malondialdehyde: Malondialdehyde levels were estimated according to the corrected thiobarbituric acid method $^{12}$. Two milliliters of extraction solution and $3 \mathrm{~mL}$ $0.5 \%$ thiobarbituric acid including $5 \%$ trichloroacetic acid were mixed vigorously. The mixture was heated at $95^{\circ} \mathrm{C}$ in a constant temperature water bath for $0.5 \mathrm{~h}$ and then cooled in ice to room temperature. After centrifuging at 5000 gn for 15 min, the supernatant was detected at 450, 532 and $600 \mathrm{~nm}$. The concentration of malondialdehyde was determined using the formula:

CMDA $\left(\mu \mathrm{mol} \mathrm{mL} \mathrm{m}^{-1}\right)=6.45 \times\left(\mathrm{D}_{532}-\mathrm{D}_{600}\right)-0.56 \times \mathrm{D}_{450}$ where $\mathrm{D}_{450}, \mathrm{D}_{532}$ and $\mathrm{D}_{600}$ are the absorbencies at 450,532 and $600 \mathrm{~nm}$, respectively.

Fractional extraction for the interfering substances: The extraction of malondialdehyde was performed using the thiobarbituric acid method. To separate the interfering substances, equal volumes of petroleum ether, ether, ethyl acetate and $n$-butyl alcohol were successively used as extracting solvents. Products of interfering substances in partial extracts that reacted with thiobarbituric acid were detected and scanned by absorption spectroscopy at $400-700 \mathrm{~nm}$.

GC-MS conditions: The ether phase extracted from golden privet and paper mulberry leaves was evaporated to dryness with nitrogen. Then the dried sample was re-dissolved using $2 \mathrm{~mL}$ ether for GC-MS analysis. Auto system Shimadzu QP 2010 GC-MS was used to identify the interfering substances. The temperature programming of GC separation was as follows: initial oven temperature was set at $150{ }^{\circ} \mathrm{C}$ and maintained at that temperature for $2 \mathrm{~min}$, then raised to $210^{\circ} \mathrm{C}$ at a ramp of $4{ }^{\circ} \mathrm{C} / \mathrm{min}$ and maintained for $2 \mathrm{~min}$ and to $280{ }^{\circ} \mathrm{C}$ at $5{ }^{\circ} \mathrm{C} \mathrm{min}^{-1}$ and maintained for $7 \mathrm{~min}$. Nitrogen was used as carrier gas with column head pressure at $12.26 \mathrm{kPa}$ in constant pressure mode. The injection volume was $2 \mu \mathrm{L}$, the programming split/splitless injection temperature was set at $280^{\circ} \mathrm{C}$ with a split ratio of 5:1 and the electron capture detector was set at $300{ }^{\circ} \mathrm{C}$. The mass spectrometer was operated at $200^{\circ} \mathrm{C}$ in electron impact mode $(70 \mathrm{eV})$, scanning from $\mathrm{m} / \mathrm{z}, 40$ to 600 in $0.3 \mathrm{~s}$ with a $0.2 \mathrm{~s}$ interval time of the scan; the temperature of the GC-MS interface was $300{ }^{\circ} \mathrm{C}$ and the voltage of the photoelectric multiplier tube was $200 \mathrm{~V}$. Mass spectra identification was carried out by comparing with the NIST 107 (National Institute of Standards and Technology, Gaithersburg, USA) and Wiley 6.0 (Wiley, New York, NY, USA) mass spectral libraries.

Statistical analysis: Data were expressed as the mean \pm SD. The statistical significance of differences between groups was determined with Student's $t$-test using SPSS software (Chicago, IL, USA). Values of $\mathrm{p}<0.05$ were considered statistically significant.

\section{RESULTS AND DISCUSSION}

Effect of malondialdehyde extraction with two different solutions: The extraction solutions used were $0.05 \mathrm{~mol}$ $\mathrm{L}^{-1} \mathrm{pH} 7.8$ phosphate buffer and $5 \%$ trichloroacetic acid solutions. To compare the extraction efficiency with different solutions, malondialdehyde concentrations were measured by the thiobarbituric acid method in 12 different plant species. The malondialdehyde concentrations obtained from the two different extraction solutions differed significantly $(P=0.031)$ from each other (Table-1). The malondialdehyde concentrations with $5 \%$ trichloroacetic acid as the extraction solution were on average $32 \%$ higher than those with phosphate buffer as the extraction solution. The solutions extracted with $5 \%$ trichloroacetic acid were clearer than those extracted with phosphate buffer (data not shown). The malondialdehyde concentrations in senescent leaves were higher than those in young or mature leaves and the difference between them was significant $(\mathrm{P}=0.025)$, except for amaranth and golden privet. The average concentration in senescent leaves was $5.41 \mu \mathrm{mol}$ $\mathrm{g}^{-1}$ fresh weight, whereas in mature leaves it was $3.90 \mu \mathrm{mol} \mathrm{g}{ }^{-1}$ fresh weight. These data indicate that the thiobarbituric acid method with trichloroacetic acid extraction solution was appropriate for analyzing malondialdehyde concentrations in most plants tested. 


\begin{tabular}{|c|c|c|c|c|}
\hline \multicolumn{5}{|c|}{$\begin{array}{c}\text { TABLE-1 } \\
\text { COMPARISON OF MDA EXTRACTION EFFECT BY TWO DIFFERENT SOLUTIONS }\end{array}$} \\
\hline \multirow{3}{*}{ Plant names } & \multicolumn{4}{|c|}{ MDA concentration ( $\mu \mathrm{mol} \mathrm{g}{ }^{-1}$ fresh weight) } \\
\hline & \multicolumn{2}{|c|}{$\mathrm{PB}$} & \multicolumn{2}{|c|}{ TCA } \\
\hline & Functional leave & Senescent leave & Functional leave & Senescent leave \\
\hline Multiflora rose & $4.45 \pm 0.99$ & $4.99 \pm 1.00$ & $8.10 \pm 1.03$ & $8.38 \pm 1.21$ \\
\hline Bamboo & $2.49 \pm 0.89$ & $3.61 \pm 0.89$ & $5.67 \pm 0.96$ & $7.68 \pm 0.94$ \\
\hline Japan arrowwood & $3.44 \pm 1.07$ & $1.21 \pm 0.77$ & $4.41 \pm 1.09$ & $5.44 \pm 0.66$ \\
\hline Alligator weed & $1.22 \pm 0.22$ & $1.45 \pm 0.19$ & $1.79 \pm 0.69$ & $2.15 \pm 0.19$ \\
\hline Green bristlegass & $2.34 \pm 0.20$ & $3.35 \pm 0.94$ & $2.33 \pm 1.00$ & $2.67 \pm 0.34$ \\
\hline Peach plant & $1.09 \pm 0.77$ & $2.76 \pm 0.97$ & $5.45 \pm 0.85$ & $4.13 \pm 0.88$ \\
\hline Amaranth & $1.67 \pm 0.91$ & $1.51 \pm 0.36$ & $0.83 \pm 0.13$ & $0.60 \pm 0.59$ \\
\hline Chinese redbud & $4.70 \pm 1.08$ & $4.88 \pm 0.88$ & $4.64 \pm 0.66$ & $6.12 \pm 0.98$ \\
\hline Golden privet & $-0.69 \pm 0.19$ & $-2.26 \pm 0.34$ & $-5.32 \pm 0.91$ & $-18.74 \pm 1.99$ \\
\hline Redflowered loropetalum & $5.63 \pm 1.11$ & $9.76 \pm 0.93$ & $2.28 \pm 0.83$ & $12.9 \pm 0.92$ \\
\hline Paper mulberry & $2.09 \pm 0.35$ & $5.38 \pm 0.99$ & $4.09 \pm 1.92$ & $4.11 \pm 1.00$ \\
\hline Tea plant & $2.45 \pm 0.11$ & $4.07 \pm 0.54$ & $3.28 \pm 0.97$ & $4.31 \pm 0.90$ \\
\hline
\end{tabular}

The solution extracted from young leaves of amaranth was redder than the solution from senescent leaves (data not shown). We suggest that the anthocyanins in amaranth leaves may be the main interfering substances that influence the accuracy of the thiobarbituric acid method; amaranth leaves have a higher anthocyanin content than leaves of other species. Unexpectedly, the malondialdehyde concentrations in golden privet leaves as measured by this method were negative and there was little red residue in the solutions extracted from these leaves. The solutions of these samples had higher absorption at $600 \mathrm{~nm}$ than at $532 \mathrm{~nm}$ (Fig. 2C).

Fractional extraction for interfering substances: To further clarify the reason for the apparent negative malondialdehyde concentrations detected by the thiobarbituric acid method in golden privet leaves, the interfering substances were separated by successive liquid-liquid extraction. Paper mulberry leaves were used as control material. Petroleum ether, ether, ethyl acetate and $n$-butyl alcohol were used as extraction solvents. Compared with the red products of malondialdehyde (solution extracted from paper mulberry) following reaction with thiobarbituric acid, the products of the interfering substances (solution extracted from golden privet) following reaction with thiobarbituric acid appeared dark green (Fig. 2A and B). The extraction results show that the concentration of the dark green interfering substance was highest in the ether phase and lowest in the ethyl acetate phase (Fig. 2A), whereas the red product of control material (paper mulberry) was highest in the water phase and lowest in the ether phase (Fig. 2B).

The absorption spectroscopy scanning results showed that the dark green substance had a characteristic absorption peak at $615 \mathrm{~nm}$, whereas the red product of the control had a characteristic peak at $500 \mathrm{~nm}$ (Fig. 2C). Detection of the interfering substance in different tissues showed that this substance was mainly found in leaves but was almost undetectable in the stems and roots (Fig. 2D).

GC-MS analysis: To determine the structure of these malondialdehyde-interfering compounds, the ether phases extracted from the golden privet leaves and stems were analyzed by GC-MS (Fig. 3). Compared with the gas chromatogram of stems, the gas chromatogram of the ether phase of golden privet leaves showed a few different peaks, such as peaks $8,9,10$ and 11 . The analysis of mass spectrum predicted that peaks 8 , 9, 10 and 11 were similar to diethyl hydroxybutanedioate (similarity: $94 \%$ ), 3,4-dimethoxybenzoic acid (72\%), quinuclidine-2-carboxylic acid, 2,3-dehydro-3-amino-ethyl ester $(63 \%)$ and tyramine, $N$-formyl-2-(4-hydroxyphenyl) ethylformamide (82\%), respectively.

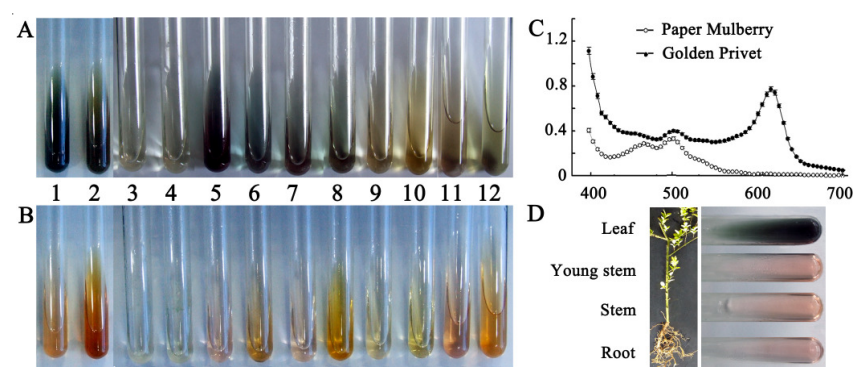

Fig. 2. Separation of the interfering substances by successive liquid-liquid extraction in golden privet and paper mulberry leaves. A: solutions extracted from golden privet; B: solutions extracted from paper mulberry; C: visible light spectra of ether extract solution; D: the accumulation of interfering substances in the different tissues of golden privet. MDA solutions were successively liquid-liquid extracted by: 1,2 $5 \%$ TCA; 3,4 petroleum ether; 5,6 ether; 7,8 ethyl acetate; 9,10 n-butyl alcohol. 11,12 show the water phase. For each solution, odd numbers indicate extraction from functional leaves and even numbers indicate extraction from senescent leaves

The rationale and methodology of the thiobarbituric acid reactive substances assay have been discussed in detail elsewhere ${ }^{10,11}$. The method has been rightly criticized for low specificity and artifact formation ${ }^{13-15}$. In recent years, several HPLC-based thiobarbituric acid assays have been developed with increased specificity ${ }^{16}$. Despite these facts, the spectrophotometric method of thiobarbituric acid remains one of the most useful and commonly used measurements of lipid peroxidation because of its simplicity.

Phosphate buffer is used as a common extraction solution for activity analysis of the enzymes in activated oxygen metabolism ${ }^{17}$. It can also be used in lipid peroxidation detection, which is very useful in rare plant studies to save 

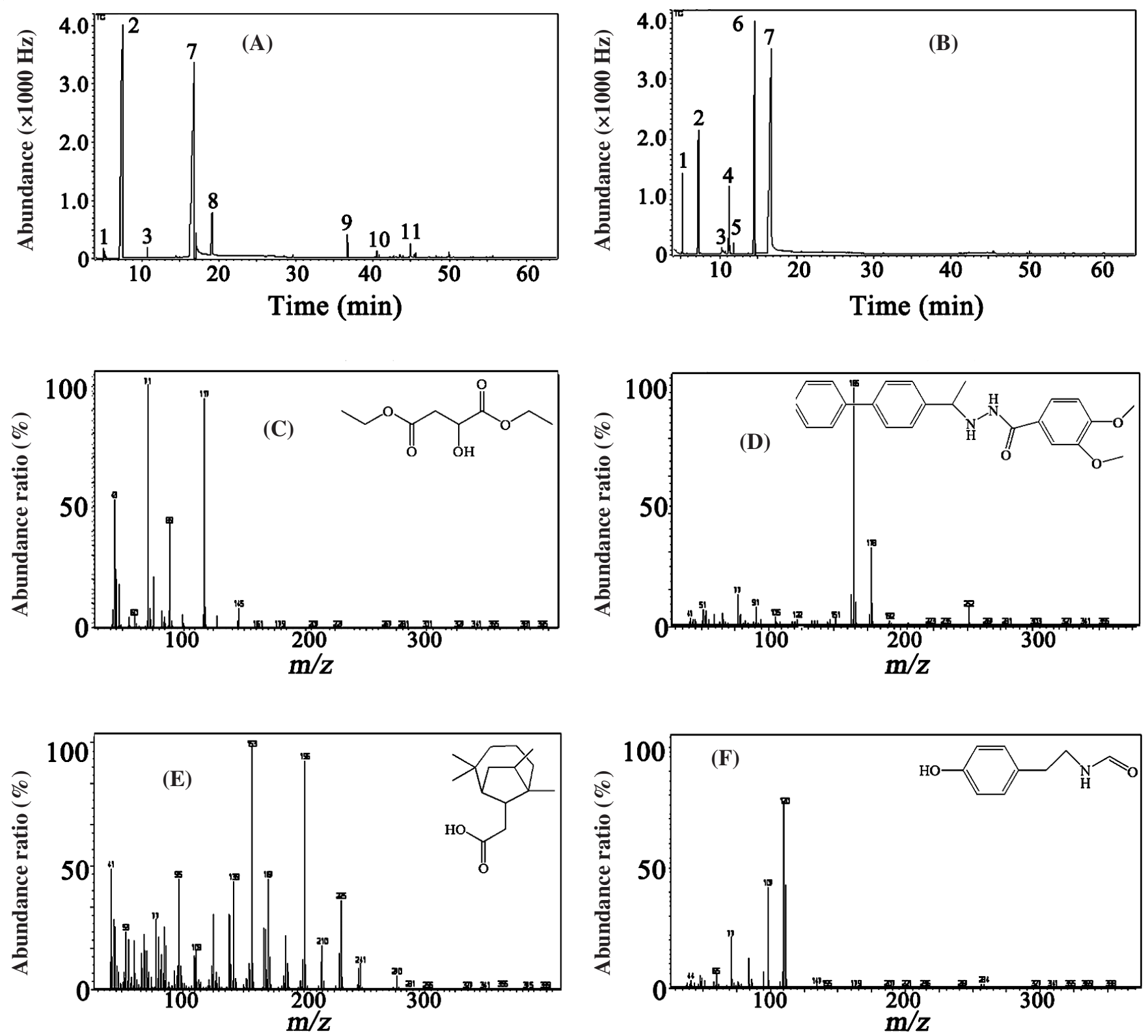

Fig. 3. GC-MS analysis of the MDA-interfering substances in golden privet leaves. Gas chromatograms of ether phase extracted from golden privet leaves (A) and stems (B) and the mass spectra of peaks 8(C), 9(D) and $10(\mathrm{E})$ and 11 (F). The mass spectra show that the peaks are most similar to the following compounds: peak 8, diethyl hydroxybutanedioate (similarity: $94 \%$ ); peak 9, 3,4-dimethoxy-benzoic acid (similarity: $74 \%$ ); peak 10, quinuclidine-2-carboxylic acid, 2,3-dehydro-3-amino-ethyl ester (similarity: $63 \%$ ); peak 11, tyramine, N-formyl-2-(4-Hydroxyphenyl) ethylformamide (similarity: $82 \%$ )

experimental material. The malondialdehyde concentrations measured by the thiobarbituric acid method with phosphate buffer and trichloroacetic acid as extraction solutions differed significantly from each other. The malondialdehyde concentrations with $5 \%$ trichloroacetic acid as the extraction solution were on average higher than those with phosphate buffer solution. In agreement with some other studies ${ }^{18,19}$, the results suggest that lipid peroxidation should be corrected for interfering compounds when measured by the thiobarbituric acid method with trichloroacetic acid as the extraction solution in most experimental plants.

Unexpectedly, an interesting result was detected that in golden privet leaves the malondialdehyde concentration was negative when measured by this method. Golden privet, also known as golden vicary privet or golden Ligustrum, is a vase- shaped shrub often used for shrub borders and hedging. Leaves of golden privet are golden yellow and lance-shaped. To further clarify the reason for the negative malondialdehyde concentrations acquired by the thiobarbituric acid method in golden privet leaves, the interfering substance was separated by successive liquid-liquid extraction. The results indicated that the interfering substances were highly lipid soluble and were present at the highest levels in the leaves and almost undetectable in stems and roots.

The product of malondialdehyde following reaction with thiobarbituric acid was measured at $532 \mathrm{~nm}$ with $600 \mathrm{~nm}$ as the reference wavelength. The latter wavelength is used merely for baseline correction ${ }^{16}$. The products of the interfering substances following reaction with thiobarbituric acid had a characteristic absorption peak at $615 \mathrm{~nm}$, which is the reason 
for the negative malondialdehyde concentrations measured by the thiobarbituric acid method in golden privet leaves.

Reaction with thiobarbituric acid yielding coloured derivatives is a characteristic of conjugated aldehydes. Futterman and Saslaw $^{20}$ found that vitamin A aldehyde reacts with aqueous thiobarbituric acid to yield a black derivative, which imparts various colours to different organic solvents. Our GC-MS data indicated that a few different substances found in the ether phase of golden privet leaves, in which peak 11 ( similar with $\mathrm{N}$-formyl-2-(4-hydroxyphenyl) ethylformamide) might be one of aldehyde compounds.

\section{Conclusion}

In conclusion, comparison of the malondialdehyde extraction rate between two commonly used solutions, phosphate buffer and trichloroacetic acid, suggested that lipid peroxidation should be corrected for interfering compounds when measured by the thiobarbituric acid method with trichloroacetic acid as extraction solution in most experimental plants. However, the thiobarbituric acid method is not suitable for measuring the malondialdehyde concentration in golden privet (Ligustrum $\times$ vicaryi) leaves, because of the existence of interfering substances. The extraction results indicate that the interfering substance was highly lipid soluble and was present at the highest levels in the leaves. Absorption spectroscopy scanning results showed that the products of the interfering substance reacted with thiobarbituric acid had a characteristic absorption peak at $615 \mathrm{~nm}$. The comparison of GC-MS profiles indicated that a few different substances were found in golden privet leaves, in which the peak 11 might be one of aldehyde compounds, similar with $N$-formyl-2-(4-Hydroxyphenyl) ethylformamide. Further exploration and a more detailed explanation of the interfering substances will be carried out in a future study.

\section{ACKNOWLEDGEMENTS}

This work was supported by the National Natural Science Foundation of China (Nos. 31000314, 31170647 and 31170282), the Natural Science Foundation of Anhui Department of Education (no. KJ2010A117).

\section{REFERENCES}

1. S. Lim, Y.H. Kim, S.H. Kim, S.Y. Kwon, H.S. Lee, J.S. Kim, K.Y. Cho, K.Y. Paek and S.S. Kwak, Mol. Breeding, 19, 227 (2007).

2. M. Khalvati, B. Bartha, A. Dupigny and P. Schröder, J. Soils Sediments, 10, 54 (2010).

3. L.R. Gutha and A.R. Reddy, Plant Mol. Biol., 68, 533 (2008).

4. R.D. Mot, G. Schoofs and I. Nagy, Arch. Microbiol., 188, 257 (2007).

5. G.V. Novitskaya, T.K. Kocheshkova, T.V. Feofilaktova and Y.I. Novitskii, Russian J. Plant Physiol., 51, 361 (2004).

6. K. Shah, R.G. Kumar, S. Verma and R.S. Dubey, Plant Sci., 161, 1135 (2001).

7. J.A. Anderson, Hort Sci., 30, 196 (1995).

8. R.L. Shewfelt and A.C. Purvis, Hort Sci., 30, 213 (1995).

9. T. Auer, G.A. Khoschsorur, H. Rabl, F. Iberer, B. Petutschnigg, A. Wasler and K.H. Tscheliessnigg, Transplant Proc., 27, 2749 (1995).

10. V. Nair and G.A. Turner, Lipids, 19, 804 (1984).

11. D. Jardine, M. Antolovich, P.D. Prenzler and K. Robards, J. Agric. Food Chem., 50, 1720 (2002).

12. D.M. Hodges, J.M. Delong, C.F. Forney and R.K. Prange, Planta, 207, 604 (1999).

13. D.R. Janero and B. Burghardt, Lipids, 23, 452 (1988).

14. J.A. Knight, R.K. Pieper and L. McClellan, Clin. Chem., 34, 2433 (1988).

15. M.W. Davey, E. Stals, B. Panis, J. Keulemans and R.L. Swennen, Anal. Biochem., 347, 201 (2005).

16. R. Parussini and C. Godinot, Free Radic. Res. Commun., 2, 93 (1986).

17. L. Jens, Clin. Chem., 47, 1725 (2001).

18. O.B. Blokhina, K.V. Fagerstedt and T.V. Chirkova, Physiol. Plant, 105, 625 (1999).

19. Z.Y. Du and W.J. Bramlage, J. Agric. Food Chem., 40, 1566 (1992).

20. S. Futterman and L.D. Saslaw, J. Biol. Chem., 236, 1652 (1961). 